\title{
Pruebas de marcadores tumorales en el cáncer de vejiga
}

\author{
G. Feil, A. Stenzl \\ Departamento de Urología. Universidad Eberhard Karl. Tübingen. Alemania.
}

Actas Urol Esp 2006; 30 (1): 38-45

\begin{abstract}
RESUMEN
PRUEBAS DE MARCADORES TUMORALES EN EL CÁNCER DE VEJIGA

El patrón de referencia para detectar un cáncer de vejiga es la cistoscopia, que puede llegar a identificar casi todas las lesiones papilares y sésiles. Es, sin embargo, un procedimiento invasivo que causa molestias a los pacientes. La citología urinaria es el marcador no invasivo estándar con una especificidad muy alta, pero con baja sensibilidad negativa para los tumores de vejiga Ta, G1 y T1. Para mejorar la detección precoz del cáncer de vejiga y para monitorizar la respuesta al tratamiento y la recurrencia tumoral, la opción serían los marcadores tumorales vesicales. Una prueba ideal para el cáncer de vejiga debería tener la posibilidad de sustituir o retrasar la cistoscopia en el seguimiento de los pacientes con cáncer de vejiga. En los últimos años, la FDA aprobó las pruebas de marcadores tumorales no invasivas ImmunoCyt / uCyt+, BTA TRAK, BTA stat, NMP22, NMP22 BladderChek y UroVysion que hemos investigado. Las pruebas demostraron mayor sensibilidad para el diagnóstico del cáncer de vejiga en comparación con la citología urinaria. En conjunto, la sensibilidad media y la especificidad media fue del 64-80\% y del 71-95\%, y los valores predictivos positivo y negativo medios para detectar los procesos malignos fueron del 49-84\% y del 79-95\%, respectivamente. Los ensayos BTA TRAK, BTA stat, NMP22 y NMP22 BladderChek están limitados por el número de resultados falsos positivos en pacientes con enfermedades urológicas benignas, por ejemplo hematuria, urocistitis, cálculos renales o infecciones de las vías urinarias. Debido a su baja especificidad BTA TRAK, BTA stat, NMP22 y NMP22 BladderChek no deben usarse sin descartar primero la presencia de una enfermedad genitourinaria benigna o maligna aparte del cáncer de vejiga. A excepción de UroVysion que alcanza un 80\% de sensibilidad y un 94\% de especificidad, ninguna de estas pruebas no invasivas ha puesto de manifiesto una sensibilidad y especificidad elevadas al mismo tiempo, que es la principal demanda que debe hacerse a un marcador tumoral ideal. Una sensibilidad insuficiente junto con una especificidad limitada no permiten sustituir la cistoscopia en el diagnóstico del cáncer de vejiga ni tomar decisiones terapéuticas basándose en un resultado de prueba positivo.
\end{abstract}

Palabras clave: Cáncer de vejiga. Marcadores urinarios. Detección precoz.

\section{ABSTRACT \\ TUMOR MARKER TESTS IN BLADDER CANCER}

The gold standard for detecting bladder cancer is cystoscopy which identifies nearly all papillary and sessile lesions. However, it is an invasive procedure causing some discomfort for patients. Urine cytology is the standard non-invasive marker with very high specificity, but unfavourable poor sensitivity for Ta, G1, and T1 bladder tumors. To improve early detection of bladder cancer as well as to monitor treatment response and tumor recurrence, bladder tumor markers are eligible. An ideal bladder cancer test would have the potential to replace or delay cystoscopy in the follow-up of bladder cancer patients. In recent years, the FDA approved non-invasive tumor marker tests ImmunoCyt / uCyt+, BTA TRAK, BTA stat, NMP22, NMP22 BladderChek, and UroVysion have been investigated. The tests demonstrated higher sensitivity for diagnosis of bladder cancer compared to urine cytology. Overall, the mean sensitivity and mean specificity was $64-80 \%$ and $71-95 \%$ and the mean positive and negative predictive values to detect malignancy were $49-84 \%$ and $79-95 \%$, respectively. BTA TRAK, BTA stat, NMP22, and NMP22 BladderChek assays are limited by false-positive results in patients with benign urological diseases such as hematuria, urocystitis, renal calculi or urinary tract infections. Due to low specificity BTA TRAK, BTA stat, NMP22, and NMP22 BladderChek should not be used without first ruling out benign or malignant genitourinary disease other than bladder cancer. With the exception of UroVysion achieving $80 \%$ sensitivity and $94 \%$ specificity, none of these non-invasive tests revealed a high sensitivity and specificity at the same time, which is a main demand to be made on an ideal tumor marker. Insufficient sensitivity along with limited specificity does not allow replacing cystoscopy in diagnosis of bladder cancer or treatment decisions based on a positive test result.

Keywords: Bladder cancer. Urine markers. Early detection. 
$\mathrm{E}$ 1 cáncer de vejiga es el cuarto tipo más común de cáncer en los varones y el octavo más común en las mujeres. Cada año en los Estados Unidos y en Alemania se diagnostican más de 50.000 y 24.000 nuevos cánceres de vejiga, respectivamente ${ }^{1,2}$. Los pacientes con cáncer de vejiga reciben tratamiento por su microhematuria indolora o hematuria intensa en ausencia de infección de las vías urinarias y/o síntomas de vaciado irritativo.

El método de referencia para detectar el cáncer de vejiga es la cistoscopia. La cistoscopia es una prueba fiable que identifica casi todas las lesiones papilares y sésiles. Sin embargo, se trata de un procedimiento invasivo que causa cierto malestar en los pacientes. En el momento del diagnóstico inicial el 65-70\% de los cánceres de vejiga están confinados en el urotelio (Ta, Tis) o invaden la lámina propia (T1) y pueden tratarse con resección endoscópica y terapia intravesical. El 30-35\% de los cánceres de vejiga recién diagnosticados han invadido el músculo intravesical (T2-T4) $^{3-5}$. Los tumores Ta, de grado 1 y grado 2 recurren en el 30-70\% de los casos y progresan a enfermedad invasiva del músculo en menos del $7 \%$ de los pacientes. Por el contrario, los tumores de estadio $\mathrm{T} 1$ de grado alto tienen un porcentaje progresión de 30-50\%3-5. El elevado porcentaje de recurrencia y el riesgo de progresión hacen necesario un seguimiento cuidadoso a intervalos regulares tras el tratamiento de cáncer de vejiga. Los protocolos de seguimiento incluyen cistoscopia cada 3 meses durante 1-2 años, cada 6 meses durante otros 2-3 años y posteriormente anualmente.

Para mejorar la detección precoz del cáncer de vejiga, así como para controlar la respuesta al tratamiento y la recurrencia del tumor, los marcadores tumorales son críticos. Una prueba ideal para detectar el cáncer de vejiga sería una prueba que fuese no invasiva, muy sensible y específica, de coste asequible, fácil de realizar y con unos resultados altamente reproducibles ${ }^{6}$. La validez de una prueba de marcadores tumorales puede expresarse como sensibilidad, especificidad, valor predictivo positivo (VPP) y valor predictivo negativo (VPN). La sensibilidad y la especificidad son términos estadísticos que se refieren a la probabilidad de identificación de aquellos con o sin el trastorno (cáncer de vejiga). El VPP y el VPN son términos que indican la probabilidad de la utilidad de una prueba a la hora de identificar verdaderos positivos y verdaderos negativos. Las definiciones se dan en la Fig. 1. Un marcador tumoral exacto también tendría el potencial de sustituir o retrasar la cistoscopia en el seguimiento de los pacientes con cáncer de vejiga. Si la prueba para un marcador del cáncer de vejiga cumpliese estos criterios, esto mejoraría la calidad de vida de los pacientes y reduciría el coste de la supervivencia al sustituir una prueba cara por un procedimiento más barato ${ }^{7}$. En los últimos años, la FDA ha aprobado varias pruebas de marcadores tumorales no invasivas. En este artículo se resume la bibliografía existente sobre las pruebas comercializadas para el diagnóstico del cáncer de vejiga ImmunoCyt/uCyt+, BTA TRAK, BTA stat, NMP22, NMP22 BladderChek y UroVysion.

\section{MARCADORES TUMORALES DE VEJIGA Citología urinaria}

Actualmente la citología urinaria es el marcador no invasivo de referencia. En una revisión reciente de la literatura, la especificidad de la citología oscilaba desde el 83\% hasta el 99,7\%, con una media \pm DE de $99 \%$ y la sensibilidad desde el 20 hasta el 53\% con una media \pm DE de $34 \%{ }^{8}$. La falta de sensibilidad del $15 \%$ en los cánceres de vejiga de tipo Ta y G1 y del 46\% en los tumores T1 es uno de sus aspectos negativos. Otras revisiones de los estudios publicados han mostrado resultados similares con una sensibilidad global del 3549\% y una especificidad del 90-95\% $\%^{7,9}$. Debido a

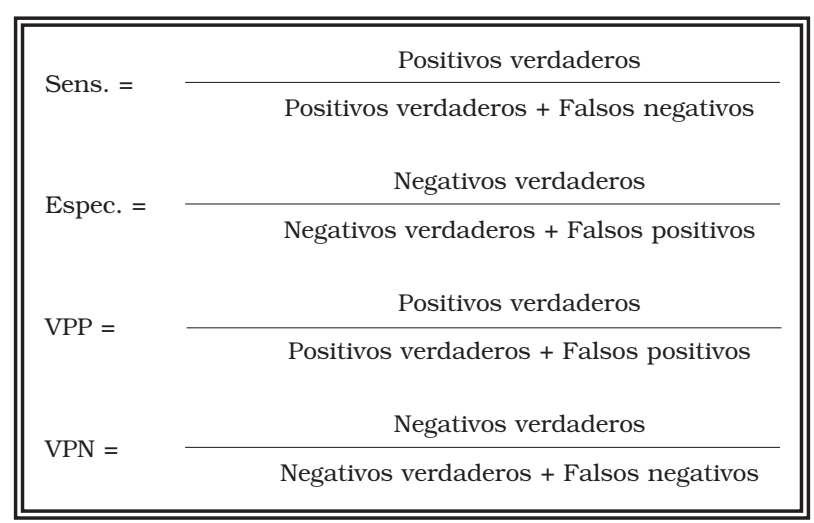

FIGURA 1: Definición de sensibilidad (Sens.), especificidad (Espec.), valor predictivo positivo (VPP) y valor predictivo negativo (VPN). 
la baja sensibilidad diagnóstica en el caso de los cánceres de vejiga de bajo grado, la citología no puede sustituir a la cistoscopia. Además, la citología es subjetiva y requiere un citopatólogo muy entrenado.

\section{Prueba ImmunoCyt / uCyt+}

El ensayo ImmunoCyt / uCyt+ (Diagnocure Inc., Québec, Canadá) es un ensayo de fluorescencia inmunocitológica en combinación con la citología urinaria de vaciado. Su evaluación requiere un citopatólogo experimentado. El ensayo identifica dos mucinas diferentes y un antígeno carcinoembrioario (CEA) glucosilado de alto peso molecular presente en las células tumorales que tienen origen en el epitelio de transición. Las mucinas y el CEA están unidos a dos anticuerpos monoclonales anti-mucina M344 y LDQ10 y al anticuerpo monoclonal anti-CEA 19A211 10,11 los cuales están marcados con FITC y Rojo de Texas, respectivamente. El ensayo ImmunoCyt / uCyt+ promete una mayor sensibilidad diagnóstica que la citologia urinaria. El ImmunoCyt / uCyt+ ha demostrado (Tabla 1) tener una sensibilidad global del 73\% (intervalo 39-100\%) y una especificidad global del $80 \%$ (intervalo $69-95 \%)^{12-20}$. Los valores predictivos positivo y negativo (Tabla 7) fueron del 52\% (intervalo 39-63\%) y 89\% (intervalo 81-97\%), respectivamente ${ }^{12,13,17,19}$. En el primer estudio de Fradet et al. ${ }^{20}$ realizado en 198 pacientes con cáncer de vejiga, ImmunoCyt / uCyt+ mostró una sensibilidad global del 90\%; la sensibilidad global de la citologia urinaria fue del $44 \%$. La evaluación combinada de ImmunoCyt / uCyt+ y la citología indicó un 95\% de sensibilidad. La especificidad de ImmunoCyt / uCyt+ solo y en combinación con la citología en 102 voluntarios asintomáticos fue del 77\%, mientras que la citología sola tuvo un $100 \%$ de especificidad. Estos datos se confirmaron en el estudio realizado por Mian et al. ${ }^{19}$ que diagnosticaron 264 pacientes consecutivos incluyendo 79 con carcinoma de células de transición de las vías urinarias. La sensibilidad global de ImmunoCyt / uCyt+, la citología urinaria y ambas pruebas combinadas fue del $86 \%, 47 \%$ y $90 \%$, respectivamente. La especificidad de ImmunoCyt / uCyt+ solo y en combinación con la citología llegó hasta el 79\%. La citología sola tuvo una especificidad del $98 \%$. En nuestra serie de 121 pacientes, entre los que se contaban 11 carcinomas pTaG1-2, 8 pT1G2-3 y 7 pT2G2-3 se observó una sensibilidad del 39\% con ImmunoCyt / uCyt+ y del 35\% en la citología. La sensibilidad combinada fue del $54 \%$. La especificidad de ImmunoCyt / uCyt+ y de la citología fue $84 \%$ y $92 \%$, respectivamente. La combinación de ambas pruebas reveló un $82 \%$ de especificidad ${ }^{15}$. Estos estudios han demostrado una mayor sensibilidad para ImmunoCyt / uCyt+ en comparación con la citologia urinaria. ImmunoCyt / uCyt+ no puede reemplazar a la citoscopia, pero puede resultar útil como complemento a la citología en el manejo del cáncer de vejiga.

\section{Ensayo BTA TRAK y ensayo BTA stat}

Las pruebas BTA TRAK y BTA stat (Bard Diagnostic Sciences Inc., Redmond, WA, EE.UU.) son inmunoensayos enzimáticos destinados a la medición de la proteína relacionada con el factor $\mathrm{H}$ del complemento humano (hCFHrp) en orina utilizando los dos anticuerpos monoclonales X13.2 y X52.1 ${ }^{21,22}$. Las células tumorales de la vejiga son hCFHrp que se liberan en la orina, presumiblemente para protegerse de ser destruidas por el sistema del complemento ${ }^{23}$. El BTA TRAK es un ensayo cuantitativo, mientras que el BTA stat es un ensayo cualitativo.
Tabla 1

Sensibilidad y especificidad de la prueba ImmunoCy/uCyt+.

\begin{tabular}{lccc}
\hline Bibliografia & \% Sensibilidad & \% Especificidad & No de pacientes \\
\hline Toma et al. $2004^{12}$ & 78 & 74 & 126 \\
Pfister et al. $2003^{13}$ & 70 & 84 & 694 \\
Drapier et al. $2003^{14}$ & 69 & 82 & 92 \\
Feil et al. $2003^{15}$ & 39 & 84 & 121 \\
Lodde et al. $2001^{16}$ & 75 & 95 & 37 \\
Vriesema et al. $2001^{17}$ & 50 & 73 & 104 \\
Olsson et al. $2001^{18}$ & 100 & 69 & 121 \\
Mian et al. $1999^{19}$ & 86 & 79 & 264 \\
Fradet et al. $1997^{20}$ & 90 & 77 & 300 \\
Media & $\mathbf{7 3}$ & $\mathbf{8 0}$ & $\mathbf{2 0 7}$ \\
\hline
\end{tabular}


En varios estudios ${ }^{24-31}$ la sensibilidad global del ensayo BTA TRAK fue del 64\% (intervalo 56$72 \%)$ y la especificidad global del 71\% (51-95\%) (Tabla 2). La sensibilidad media para el ensayo BTA stat (Tabla 3) fue del $72 \%$ (intervalo 57$100 \%$ ) y la especificidad media del $74 \%$ (intervalo 64-93\%) ${ }^{32-41}$. Los valores predictivos diagnósticos positivos y negativos de los ensayos BTA (Tabla 7) oscilaron de 35-68\% y 70-95\%, respectivamente ${ }^{26,32,36,40}$. Sarosdy et al. ${ }^{41}$ evaluaron la prueba BTA stat en la detección del cáncer de vejiga recurrente y consiguieron una sensibilidad del 67\% (147/220). Para aquellos pacientes de los que se disponen resultados citológicos previos, la citología tuvo una sensibilidad del $23 \%$ y la prueba BTA stat del 58\% para la detección del

Tabla 2

Sensibilidad y especificidad de la prueba BTA TRAK.

\begin{tabular}{lccc}
\hline Bibliografia & $\begin{array}{c}\text { \% } \\
\text { Sensibilidad }\end{array}$ & $\begin{array}{c}\text { \% } \\
\text { Especificidad }\end{array}$ & $\begin{array}{c}\text { No de } \\
\text { pacientes }\end{array}$ \\
\hline Fernández Gómez et al. 2002 & 62 & 68 & 700 \\
Priolo et al. $2001^{25}$ & 63 & 63 & 518 \\
Serretta et al. $2000^{26}$ & 62 & 79 & 74 \\
Heicappell et al. 199927 & 72 & 51 & 411 \\
Mahnert et al. $1999^{28}$ & 56 & 67 & 244 \\
Thomas et al. $1999^{29}$ & 66 & 69 & 220 \\
Abbate et al. $1998^{30}$ & 62 & 95 & 107 \\
Ellis et al. $1997^{31}$ & 72 & 75 & 216 \\
Media & $\mathbf{6 4}$ & $\mathbf{7 1}$ & $\mathbf{3 1 1}$ \\
\hline
\end{tabular}

Tabla 3

Sensibilidad y especificidad de la prueba BTA stat.

\begin{tabular}{|c|c|c|c|}
\hline Bibliografia & $\begin{array}{c}\% \\
\text { Sensibilidad }\end{array}$ & $\begin{array}{c}\% \\
\text { Especificidad }\end{array}$ & $\begin{array}{c}\text { № de } \\
\text { pacientes }\end{array}$ \\
\hline Giannopoulos et al. $2001^{32}$ & 73 & 65 & 213 \\
\hline Heicappell et al. $2000^{33}$ & 63 & 93 & 354 \\
\hline Nasuti et al. $1999^{34}$ & 100 & 84 & 100 \\
\hline Ramakumar et al. $1999^{35}$ & 74 & 73 & 196 \\
\hline Sharma et al. $1999^{36}$ & 67 & 82 & 199 \\
\hline Leyh et al. $1999^{37}$ & 65 & 64 & 240 \\
\hline Sözen et al. $1999^{38}$ & 70 & 68 & 140 \\
\hline Pode et al. $1999^{39}$ & 83 & 69 & 250 \\
\hline Wiener et al. $1998^{40}$ & 57 & 68 & 291 \\
\hline Sarosdy et al. $1997^{41}$ & 67 & 72 & 220 \\
\hline Media & 72 & 74 & 220 \\
\hline
\end{tabular}

cáncer recurrente $(\mathrm{p}<0,001)$. Ellis et al. ${ }^{31}$ describieron una sensibilidad global del $72 \%(151 / 216)$ con el ensayo BTA TRAK en pacientes con cáncer de vejiga recurrente. En comparación con la citología urinaria, BTA TRAK y BTA tuvieron sensibilidades más altas pero especificidades más bajas $^{35,37,39,41}$. Sin embargo, para los tumores de bajo grado y bajo estadiaje la sensibilidad es comparable a las sensibilidades publicadas de la citología urinaria ${ }^{33}$. La especificidad de las pruebas BTA fue menor en pacientes con otro cáncer genitourinario o enfermedad genitourinaria benigna, como hematuria, inflamación de las vías urinarias o cálculos renales que en voluntarios sanos $^{36,37}$. Además, el tratamiento intravesical del bacilo de Calmette-Guerin puede reducir la especificidad en un $28 \% 39$. Las pruebas BTA son superiores a la citología urinaria de vaciado en el diagnóstico del cáncer de vejiga y a la detección del cáncer de vejiga recurrente. Debido a su baja especificidad, la prueba BTA no debería usarse sin descartar antes la presencia de una enfermedad genitourinaria benigna o maligna distinta al cáncer de vejiga.

Pruebas NMP22 y NMP22

BladderChek

NMP22 y NMP22 BladderChek (Matritech Inc., Newton, MA, EE.UU.) son pruebas destinadas a la medición de la proteína 22 de la matriz nuclear, la cual es liberada en grandes cantidades en la orina por las células tumorales apoptóticas. NMP22 es un inmunoensayo cuantitativo, mientras que NMP22 BladderChek es un análisis cuantitativo rápido que se puede realizar en la consulta antes o en el momento de la visita para hacer la citoscopia. Varios estudios ${ }^{35,40,42-}$ ${ }^{51}$ han demostrado una sensibilidad y especificidad global del 66\% (intervalo 47-89\%) y del $75 \%$ (intervalo 60-84\%), respectivamente para el ensayo NMP22 (Tabla 4). 
Tabla 4

Sensibilidad y especificidad de la prueba NMP22.

\begin{tabular}{|c|c|c|c|}
\hline Bibliografia & $\begin{array}{c}\% \\
\text { Sensibilidad }\end{array}$ & $\begin{array}{c}\% \\
\text { Especificidad }\end{array}$ & $\begin{array}{c}\mathrm{N}^{\circ} \text { de } \\
\text { pacientes }\end{array}$ \\
\hline Lekili et al. $2004^{42}$ & 53 & 83 & 78 \\
\hline Lahme et al. $2001^{43}$ & 63 & 66 & 144 \\
\hline Giannopoulos et al. $2001^{44}$ & 64 & 75 & 213 \\
\hline Ponsky et al. $2001^{45}$ & 89 & 84 & 608 \\
\hline Ramakumar et al. $1999^{35}$ & 53 & 60 & 196 \\
\hline Hughes et al. $1999^{46}$ & 47 & 79 & 107 \\
\hline Wiener et al. $1998^{40}$ & 48 & 70 & 291 \\
\hline Stampfer et al. $1998^{47}$ & 68 & 80 & 231 \\
\hline Witjes et al. $1998^{48}$ & 75 & 82 & 50 \\
\hline Landman et al. $1998^{49}$ & 81 & 77 & 47 \\
\hline Miyanaga et al. $1997^{50}$ & 81 & 64 & 300 \\
\hline Soloway et al. $1996^{51}$ & 74 & 79 & 112 \\
\hline Media & 66 & 75 & 198 \\
\hline
\end{tabular}

especificidad (Tabla 5) del NMP22 BladderChek ${ }^{52-55}$. Tomera et al. ${ }^{55}$ identificaron el 61\% (11/18) de los carcinomas de células de transición, incluido un carcinoma uretral en pacientes de alto riesgo. NMP22 BladderChek fue más valioso que la citología con un 18\% de sensibilidad. La especificidad de NMP22 BladderChek y la citología en pacientes no diagnosticados de cáncer de vejiga fue del $89 \%$ y 99\%, respectivamente. Oehr ${ }^{53}$ excluyó a los pacientes con urocistitis, cálculos, infecciones de las vías urinarias y sondas implantadas, observando una sensibilidad y especificidad del $82 \%$ y $90 \%$ en el grupo de hematuria y del $61 \%$ y $98 \%$ en el grupo de seguimiento, respectivamente. Globalmente, en el grupo de NMP22

Se observó un aumento de la sensibilidad a medida que aumentaba el estadio y el grado del tumor ${ }^{43,49}$. Soloway et al. ${ }^{51}$ controlaron el estado de la enfermedad tras la resección endoscópica del tumor y observaron un 70\% (23/33) de recurrencias. Al comparar la NMP22 urinaria y la citología urinaria de vaciado, NMP22 mostró una mayor sensibilidad en pacientes con cáncer urotelial $^{43,47,50}$. NMP22 es superior a la citología en cuanto a la detección de tumores de bajo grado, pero presenta una especificidad limitada. La observación y exclusión de lesiones benignas puede aumentar la especificidad, como han demostrado Ponsky et al. ${ }^{45}$. Tras la exclusión de los resultados falsos positivos en 608 pacientes, estos autores observaron una sensibilidad y especificidad global del 89\% y 84\%. NMP22 ha sido aprobada por la FDA para la detección del cáncer de vejiga y Yokoyama et al. 200452 puede ser útil para el diagnóstico de pacientes sintomáticos o personas con exposición ocupacional a carcinógenos conocidos como aminas aromáticas, hidrocarburos aromáticos policíclicos o disolventes clorados. Sólo se dispone de los datos preliminares sobre sensibilidad $y$
Tabla 5

Sensibilidad y especificidad de la prueba NMP22 BladderChek.

\begin{tabular}{lccc}
\hline Bibliografia & \% Sensibilidad & \% Especificidad & No de pacientes \\
\hline Yokoyama et al. $2004^{52}$ & 57 & n.e. & 51 \\
Oehr et al. $2004^{53}$ & $82^{*}, 61^{* *}$ & $98^{* * * *}$ & $212^{*}, 206^{* *}$ \\
Gutiérrez et al. $2004^{54}$ & n.e. & 93 & $23^{* * *}$ \\
Tomera et al. $2003^{55}$ & 61 & 89 & 248 \\
Media & $\mathbf{6 5}$ & $\mathbf{9 5}$ & $\mathbf{1 4 8}$ \\
\hline
\end{tabular}

**grupo de seguimiento

***tratamiento del BCG

n.e. $=$ no especificado *grupo de hematuria
BladderChek test se obtuvo un menor porcentaje de resultados falsos positivos que con el ensayo NMP22, lo que dio más valores predictivos positivos y negativos (Tabla 7) 40,42,44,45,47,48,51,53,54. La exactitud de NMP22 y NMP22 BladderChek, respectivamente, para el diagnóstico fiable de pacientes con riesgo de cáncer de vejiga deberá ser evaluada en un estudio multicéntrico prospectivo.

\section{Prueba UroVysion}

UroVysion (Abbott Laboratories Inc., Downers Grove, IL, EE.UU.) es una técnica de hibridación in situ con fluorescencia multisonda (FISH) para detectar la aneuploidia en los cromosomas 3, 7 y 
17, y la pérdida (deleción) del locus 9p21 mediante hibridación in situ con fluorescencia (FISH) en células tumorales de vejiga exfoliadas. La Tabla 6 muestra los estudios realizados con la prueba UroVysion publicados por varios grupos ${ }^{56-60}$. En general, UroVysion mejora los porcentajes de sensibilidad descritos para la citología urinaria en la detección de todos los estadios y grados del tumor con una especificidad similar. El cáncer de vejiga de grado 1 y grado 2 se detectó en $36-83 \%$ y en $76-83 \%$, respectivamente. Placer et al. ${ }^{57}$ describieron una menor sensibilidad para la citología, con un $64 \%$ globalmente, $25 \%$ para los tumores de grado $1,1,67 \%$ para los de grado $2 \mathrm{y}$ 95\% para los de grado 3 en comparación con FISH, con un $80 \%, 53 \%, 83 \%$ y $100 \%$, respectivamente. Skacel et al. ${ }^{56}$ analizaron muestras de orina de pacientes con citología sospechosa, atípica y negativa y los compararon con los datos de la biopsia. La sensibilidad fue del 100\%, 89\% y $60 \%$, respectivamente. Nueve pacientes con cito-

Tabla 6

Sensibilidad y especificidad de la prueba UroVysion.

\begin{tabular}{|c|c|c|c|c|c|c|}
\hline \multirow[t]{2}{*}{ Bibliografia } & \multicolumn{4}{|c|}{$\%$ Sensibilidad } & \multirow{2}{*}{$\begin{array}{c}\% \\
\text { Especificidad }\end{array}$} & \multirow{2}{*}{$\begin{array}{c}\text { № de } \\
\text { pacientes }\end{array}$} \\
\hline & G1 & G2 & G3 & G1-3 & & \\
\hline Skacel et al. $2003^{56}$ & 83 & 80 & 96 & 85 & 97 & 120 \\
\hline Placer et al. $2002^{57}$ & 53 & 83 & 100 & 80 & 85 & 86 \\
\hline Sarosdy et al. $2002^{58}$ & 55 & 78 & 94 & 71 & 95 & 176 \\
\hline Halling et al. $2000^{59}$ & 36 & 76 & 97 & 81 & 96 & 265 \\
\hline Media & 57 & 79 & 97 & 79 & 93 & 162 \\
\hline Bubendorf et al. $2001^{60}$ & $\begin{array}{c}\text { pTa } \\
73\end{array}$ & $\begin{array}{l}\text { pT1 } \\
100\end{array}$ & $\begin{array}{c}\text { pT2-4 } \\
100\end{array}$ & $\begin{array}{c}\text { pTa- } 4 \\
83\end{array}$ & 96 & 97 \\
\hline Media & & & & 80 & 94 & 149 \\
\hline
\end{tabular}

logía atípica y biopsia de vejiga negativa dieron FISH positivo. Sin embargo, en todas las biopsias realizadas en el plazo de 15 meses como máximo desde la fecha de análisis con FISH se detectó el carcinoma. Los resultados conseguidos con UroVysion destacan el importante papel de este ensayo en el manejo del cáncer de vejiga. UroVysion ofrece una alta sensibilidad y especificidad para detectar el carcinoma de células de transición en muestras de orina con resultados citológicos dudosos o negativos. UroVysion parece ser un marcador tumoral prometedor para la detección precoz del cáncer de vejiga recurrente y podría ser útil para la detección de personas con riesgo de cáncer de vejiga.

\section{CONCLUSIONES}

Los recientes desarrollos habidos en el campo de las pruebas de marcadores tumorales de vejiga consisten en mediciones cualitativas o cuantitativas de las proteínas urinarias o la detección de antígenos de aberraciones cromosómicas en muestras de citologia urinaria. Estas pruebas de marcadores tumorales han demostrado una mayor sensibilidad para el diagnóstico del cáncer urotelial en comparación con la citología urinaria. En general, la sensibilidad y especificidad media fue $64-80 \%$ y $71-95 \%$ y los valores predictivos positivos y negativos medios para detectar el tumor fueron $49-84 \%$ y $79-95 \%$, respectivamente (Tabla 7). Sin embargo, la sensibilidad para el cáncer de veji-

Tabla 7

Sensibilidad, especificidad, valor predictivo positivo (VPP) y negativo (VPN) de las pruebas de marcadores tumorales de vejiga.

\begin{tabular}{lccccc}
\hline Prueba & $\begin{array}{c}\text { \% Sensibilidad } \\
\text { media (intervalo) }\end{array}$ & $\begin{array}{c}\text { \% Especificidad } \\
\text { media (intervalo) }\end{array}$ & $\begin{array}{c}\text { \% PPV } \\
\text { media (intervalo) }\end{array}$ & $\begin{array}{c}\text { \% NPV } \\
\text { media (intervalo) }\end{array}$ & Bibliografia \\
\hline ImmunoCyt / uCyt+ & $73(39-100)$ & $80(69-95)$ & $52(39-63)$ & $89(81-97)$ & {$[12-20]$} \\
BTA TRAK & $64(56-72)$ & $71(51-95)$ & 45 & 88 & {$[24-31]$} \\
BTA stat & $72(57-100)$ & $74(64-93)$ & $49(35-68)$ & $81(70-95)$ & {$[32-41]$} \\
NMP22 & $66(47-89)$ & $75(60-84)$ & $55(34-74)$ & $79(65-91)$ & {$[35,40,42-51]$} \\
NMP22 BladderChek & $65(57-82)$ & $95(89-98)$ & $84(82-85)$ & $95(93-98)$ & {$[52-55]$} \\
UroVysion & $80(71-85)$ & $94(85-97)$ & 53 & 81 & {$[56-60]$} \\
\hline
\end{tabular}


ga Ta de grado 1 fue deficiente en las pruebas que detectan las proteínas liberadas en la orina por las células tumorales. Los ensayos BTA TRAK, BTA stat, NMP22 y NMP22 BladderChek están limitados por resultados falsos positivos en pacientes con enfermedad urológica benigna, como hematuria, urocistitis, cálculos renales o infecciones urinarias, así como en pacientes con sondas implantadas o manipulación intravesical en las semanas anteriores. Debido a la baja especificidad, BTA TRAK, BTA stat, NMP22 y NMP22 BladderChek no deberían utilizarse sin haberse descartado primero la presencia de una enfermedad genitourinaria benigna o maligna diferente al cáncer de vejiga. La insuficiente sensibilidad junto con la limitada especificidad no permiten sustituir la cistoscopia en el diagnóstico del cáncer de vejiga o las decisiones de tratamiento basadas en un resultado positivo de la prueba. A excepción de UroVysion que consigue un 80\% de sensibilidad y un 94\% de especificidad, ninguna de estas pruebas no invasivas ha revelado una elevada sensibilidad y especificidad simultáneas, lo cual sería uno de los criterios principales de un marcador tumoral ideal. Además, UroVysion no está limitado por criterios de exclusión.

\section{REFERENCIAS}

1. Boring CC, Squires TS, Tong T, Montgomery S: Cancer statistics, 1994. CA Cancer J Clin 1994;44:7-26.

2. Arbeitsgemeinschaft Bevölkerungsbezogener Krebsregister in Deutschland (Hrsg). Krebs in Deutschland. 4. überarbeitete, aktualisierte Ausgabe, Saarbrücken, 2004.

3. Sebe P, Lebret T, Molinie V, Herve J M, Yonneau L, Lugagne P M, et al. Superficial grade G2 tumors of the bladder: recurrence, progression, prognosis. Prog Urol 2003;13:608-612.

4. Fitzpatrick JM, West AB, Butler MR, Lane V, O'Flynn JD. Superficial bladder tumors (stages pTa, grades 1 and 2): the importance of recurrent pattern following initial resection. J Urol 1986; 135:920-922.

5. Heney NM, Ahmed S, Flanagan MJ, Frable W, Corder MP, Hafermann MD, et al. Superficial bladder cancer: progression and recurrence. J Urol 1983;130:1083-1086.

6. Simon MA, Lokeshwar VB, Soloway MS. Current bladder cancer tests: unnecessary or beneficial? Crit Rev Oncol Hematol 2003;47:91-107.

7. Lokeshwar VB, Soloway MS: Current bladder tumor tests: does their projected utility fulfill clinical necessity? J Urol 2001;165:1067-1077.

8. Lotan Y, Roehrborn CG. Cost-effectiveness of a modified care protocol substituting bladder tumor markers for cystoscopy for the followup of patients with transitional cell carcinoma of the bladder: a decision analytical approach. J Urol 2002;167:75-79.

9. Konety BR, Getzenberg RH. Urine based markers of urological malignancy. J Urol 2001;165:600-611.

10. Fradet Y, Islam M, Boucher L, Parent-Vaugeois C, Tardif M. Polymorphic expression of a human superficial bladder tumor antigen defined by mouse monoclonal antibod-ies. Proc Natl Acad Sci 1987;84:7272-7231.
11. Fradet Y, LaRue H, Parent-Vaugeois C, Bergeron A, Dufour C, Boucher L, et al. Monoclonal antibody against a tumor-associated sialoglycoprotein of superficial papillary bladder tumors and cervical condylomas. Int J Cancer 1990;46:990-997.

12. Toma MI, Friedrich MG, Hautmann SH, Jakel KT, Erbersdobler A, Hellstern A, et al. Comparison of the ImmunoCyt test and urinary cytology with other urine tests in the detection and surveillance of bladder cancer. World J Urol 2004;22:145-149.

13. Pfister C, Chautard D, Devonec M, Perrin P, Chopin D, Rischmann $\mathrm{P}$, et al. Immunocyt test improves the diagnostic accuracy of urinary cytology: results of a French multicenter study. J Urol 2003; 169:921-924.

14. Drapier E, Renaudin K, Maillet F, Braud G, Laboisse C, Bouchot O. Value of the uCyt+ test for the detection and followup of bladder tumors. Prog Urol 2003;13:222-226.

15. Feil G, Zumbrägel A, Päulgen-Nelde HJ, Hennenlotter J, Maurer S, Krause S, et al. Accuracy of the ImmunoCyt assay in the diagnosis of transitional cell carcinoma of the urinary bladder. Anticancer Res 2003;23(2A):963-967.

16. Lodde M, Mian C, Wiener H, Haitel A, Pycha A, Marberger M. Detection of upper urinary tract transitional cell carcinoma with ImmunoCyt: a preliminary report. Urology 2001;58:362-366.

17. Vriesema JL, Atsma F, Kiemeney LA, Peelen WP, Witjes JA, Schalken JA. Diagnostic efficacy of the ImmunoCyt test to detect superficial bladder cancer recurrence. Urology 2001;58:367-371.

18. Olsson H, Zackrisson B. ImmunoCyt a useful method in the followup protocol for patients with urinary bladder carcinoma. Scand $\mathrm{J}$ Urol Nephrol 2001;35:280-282.

19. Mian C, Pycha A, Wiener H, Haitel A, Lodde M, Marberger M. Immunocyt: a new tool for detecting transitional cell cancer of the urinary tract. J Urol 1999;161:1486-1489.

20. Fradet Y, Campbell L, Emond J-P, Zadra J, Jewett M, Bertrand P, et al. Performance characteristics of a new monoclonal antibody test for bladder cancer: ImmunoCyt ${ }^{\mathrm{TM}}$. Cand J Urol 1997;4:400405.

21. Kinders R, Jones T, Root R, Bruce C, Murchison H, Corey M, et al. Complement factor $\mathrm{H}$ or a related protein is a marker for transitional cell cancer of the bladder. Clin Cancer Res 1998;4:2511-2520.

22. Corey MJ, Kinders RJ, Poduje CM, Bruce CL, Rowley H, Brown LG, et al. Mechanistic studies on the effect of anti-factor $\mathrm{H}$ antibodies on com-plement-mediated lysis. J Biol Chem 2000;275:1291712925.

23. Austyn JM, Wood KJ. Principles of cellular and molecular immunology. Oxford University Press, Oxford, 1993;522-554.

24. Fernández Gómez JM, García Rodríguez J, Escaf Barmadah S, Raigoso P, Rodríguez Martínez J , Allende MT, et al. Urinary BTATRAK in the follow-up of superficial transitional-cell bladder carcinoma. Arch Esp Urol 2002;55:41-49.

25. Priolo G, Gontero P, Martinasso G, Mengozzi G, Formiconi A, Pelucelli G, et al. Bladder tumor antigen assay as compared to voided urine cytology in the diagnosis of bladder cancer. Clin Chim Acta 2001;305:47-53.

26. Serretta V, Pomara G, Rizzo I, Esposito E. Urinary BTA-Stat, BTATrak and NMP22 in surveillance after TUR of recurrent superficial transitional cell carcinoma of the bladder. Eur Urol 2000;38:419425.

27. Heicappell R, Wettig IC, Schostak M, Müller M, Steiner U, Sauter $\mathrm{T}$, et al. Quantitative detection of human complement factor $\mathrm{H}$ related protein in transitional cell carcinoma of the urinary bladder. Eur Urol 1999;35:81-87.

28. Mahnert B, Tauber S, Kriegmair M, Schmitt UM, Hasholzner U, Reiter W, et al. BTA-TRAK - a useful diagnostic tool in urinary bladder cancer? Anticancer Res 1999;19:2615-2619.

29. Thomas L, Leyh H, Marberger M, Bombardieri E, Bassi P, Pagano F, et al. Multicenter trial of the quantitative BTA TRAK assay in the detection of bladder cancer. Clin Chem 1999;45:472-477. 
30. Abbate L, D'Introno A, Cardo G, Marano A, Addabbo L, Musci MD, et al. Comparison of nuclear matrix protein 22 and bladder tumor antigen in urine of patients with bladder cancer. Anticancer Res 1998; 18:3803-3805.

31. Ellis WJ, Blumenstein BA, Ishak LM, Enfield DL. Clinical evaluation of the BTA TRAK assay and comparison to voided urine cytology and the Bard BTA test in patients with recurrent bladder tumors. The Multi Center Study Group. Urology 1997;50:882-887.

32. Giannopoulos, A, Manousakas T, Gounari A, Constantinides C, Choremi-Papadopoulou H, Dimopoulos C. Comparative evaluation of the diagnostic performance of the BTA stat test, NMP 22 and urinary bladder cancer antigen for primary and recurrent bladder tumors. J Urol 2001;2:470-475.

33. Heicappell R, Muller M, Fimmers R, Miller K. Qualitative determination of urinary human complement factor H-related protein (hcfHrp) in patients with bladder cancer, healthy controls, and patients with benign urologic disease. Urol Int 2000;65:181-184.

34. Nasuti JF, Gomella LG, Ismial M, Bibbo M. Utility of the BTA stat test kit for bladder cancer screening. Diagn Cytopathol 1999;21:27-29.

35. Ramakumar S, Bhuiyan J, Besse JA, Roberts SG, Wollan PC Blute ML, et al. Comparison of screening methods in the detection of bladder cancer. J Urol 1999;161:388-394.

36. Sharma S, Zippe CD, Pandrangi L, Nelson D, Agarwal A. Exclusion criteria enhance the specificity and positive predictive value of NMP22 and BTA stat. J Urol 1999;162:53-57.

37. Leyh H, Marberger M, Conort P, Sternberg C, Pansadoro V, Pagano $\mathrm{F}$, et al. Comparison of the BTA stat test with voided urine cytology and bladder wash cytology in the diagnosis and monitoring of bladder cancer. Eur Urol 1999;35:52-56.

38. Sözen S, Biri H, Sinik Z, Küpeli B, Alkibay T, Bozkirli I Comparison of the nuclear matrix protein 22 with voided urine cytology and BTA stat test in the diagnosis of transitional cell carcinoma of the bladder. Eur Urol 1999;36:225-229.

39. Pode D, Shapiro A, Wald M, Nativ O, Laufer M, Kaver I. Noninvasive detection of bladder cancer with the BTA stat test. J Urol 1999;161:443-446.

40. Wiener HG, Mian C, Haitel A, Pycha A, Schatzl G, Marberger M. Can urine bound diagnostic tests replace cystoscopy in the management of bladder cancer? J Urol 1998;159:1876-1880.

41. Sarosdy MF, Hudson MA, Ellis WJ, Soloway MS, deVere White R, Sheinfeld J, et al. Improved detection of recurrent bladder cancer using the Bard BTA stat Test. Urology 1997; 50: 349-353

42. Lekili M, Sener E, Demir MA, Temeltas G, Muezzinoglu T, Buykusu C. Comparison of the nuclear matrix protein 22 with voided urine cytology in the diagnosis of transitional cell carcinoma of the bladder. Urol Res 2004;32:124-128.

43. Lahme S, Bichler K-H, Feil G, Krause S. Comparison of cytology and nuclear matrix protein 22 for the detection and follow-up of bladder cancer. Urol Int 2001;66:72-77.

44. Giannopoulos A, Manousakas T, Gounari A, Constantinides C, Choremi-Papadopoulou H, Dimopoulos C. Comparative evaluation of the diagnostic performance of the BTA stat test, NMP 22 and urinary bladder cancer antigen for primary and recur-rent bladder tumors. J Urol 2001:2:470-475.

45. Ponsky LE, Sharma S, Pandrangi L, Kedia S, Nelson D, Agarwal A et al. Screening and monitoring for bladder cancer: refining the use of NMP22. J Urol 2001;1:75-78.

46. Hughes JH, Katz RL, Rodríguez-Villanueva J, Kidd L, Dinney C, Grossman HB, et al. Urinary nuclear matrix protein 22 (NMP22): a diagnostic adjunct to urine cytologic examination for the detection of recurrent transitional-cell carcinoma of the bladder. Diagn Cytopathol 1999;20:285-290.
47. Stampfer DS, Carpinito GA, Rodríquez-Villanueva J, Willsey LW, Dinney CP, Grossman HB. Evaluation of NMP 22 in the detection of transitional cell carcinoma of the bladder. J Urol 1998;159:394-398.

48. Witjes JA, van der Poel HG, van Balken MR, Debruyne FM, Schalken JA. Urinary NMP22 and karyometry in the diagnosis and follow-up of patients with superficial bladder cancer. Eur Urol 1998;33(4):387-391.

49. Landman J, Chang Y, Kavaler E, Droller MJ, Liu C-S. Sensitivity and specificity of NMP-22, telomerase, and BTA in the detection of human bladder cancer. J Urol 1998;52:398-402.

50. Miyanaga N, Akaza H, Ishikawa S, Ohtani M, Noguchi R, Kawai K, Koiso K. Clinical evaluation of nuclear matrix protein 22 (NMP22) in urine as a novel marker for urothelial cancer. Eur Urol 1997;31:163-168.

51. Soloway MS, Briggman V, Carpinito GA, Chodak GW, Church PA, Lamm DL. Use of a new tumor marker, urinary NMP22, in the detection of occult or rapidly recurring transitional cell carcinoma of the urinary tract following surgical treatment. J Urol 1996;156:363-367.

52. Yokoyama T, Sekigawa R, Hayashi T, Horita S, Kanamuro T, Nonami Y, et al. The clinical efficacy of BladderChek NMP22 in urothelial cancer. Rinsho Byori 2004;52:199-203.

53. Oehr P. Non-invasive screening of urinary bladder cancer. Is NMP22 BladderChek paving the avenue? (abstract) J Urol 2004; 171 Suppl:70.

54. Gutiérrez C, Palou J, Bujons A, Iglesias JC, Juaneda B, Segarra J, et al. The detection of nuclear matrix protein 22 in the follow up of patients after endovesical treatment with BCG. (abstract) Eur Urol 2004;Suppl3(2):97.

55. Tomera K, Clark WR, Singsaas MW, Strawbridge LR. Results of screening high risk patients for urothelial cancers with a new office test NMP22 BladderChek. (abstract) J Urol 2003;169Suppl: 226-227.

56. Skacel M, Fahmy M, Brainard JA, Pettay JD, Biscotti CV, Liou LS et al. Multitarget fluorescence in situ hybridization assay detects transitional cell carcinoma in the majority of patients with bladder cancer and atypical or negative urine cytology. J Urol 2003;169: 2101-2105.

57. Placer J, Espinet B, Salido M, Sole F, Gelabert-Mas A. Clinical utility of multiprobe FISH assay in voided urine specimens for the detection of bladder cancer and its recurrences compared with urinary cytology. EurUrol 2002;42 (6):547-552.

58. Sarosdy MF, Schellhammer P, Bokinsky G, Kahn P, Chao R, Yore L. Clinical evaluation of a multi-target fluorescent in situ hybridization assay for detection of bladder cancer. $J$ Urol 2002; 168:1950-1954.

59. Halling KC, King W, Sokolova IA, Meyer RG, Burkhardt HM, Halling AC, et al. A comparison of cytology and fluorescence in situ hybridization for the detection of urothelial carcinoma. J Urol 2000;164:1768-1775

60. Bubendorf L, Grilli B, Sauter G, Mihatsch MJ, Gasser TC, Dalquen P. Multiprobe FISH for enhanced detection of bladder cancer in voided urine specimens and bladder washings. Am J Clin Pathol 2001;116:79-86.

Gerhard Feil, Ph. D.

Department of Urology

Eberhard Karls University

Hoppe-Seyler-Strasse, 3

72076 Tübingen (Alemania)

E-mail: gerhard.feil@med.uni-tuenbingen.de

(Trabajo recibido el 22 de septiembre 2005) 